\title{
Helicobacter pylori-induced STAT3 activation and signalling network in gastric cancer
}

\author{
Junhong Zhao ${ }^{1, *}$, Yujuan Dong ${ }^{1,3, *}$, Wei Kang ${ }^{2}$, Minnie Y. Go ${ }^{1}$, Joanna HM. Tong ${ }^{2}$, \\ Enders KW. Ng${ }^{3}$, Philip WY. Chiu ${ }^{3}$, Alfred SL. Cheng ${ }^{1}$, Ka Fai To², Joseph JY. Sung ${ }^{1}$, \\ Jun $Y \mathbf{u}^{1}$ \\ ${ }^{1}$ Institute of Digestive Disease and Department of Medicine and Therapeutics, State Key Laboratory of Digestive Disease, Li \\ Ka Shing Institute of Health Sciences, The Chinese University of Hong Kong, Hong Kong, China \\ ${ }^{2}$ Department of Anatomical and Cellular Pathology, The Chinese University of Hong Kong, Hong Kong, China \\ ${ }^{3}$ Department of Surgery, The Chinese University of Hong Kong, Hong Kong, China \\ * The authors contribute equally to this study. \\ Correspondence to: Jun Yu, email: junyu@cuhk.edu.hk
}

Keywords: Helicobacter pylori; STAT3; gastric carcinogenesis; molecular regulator

Received: June 24, 2014

Accepted: July 2, 2014

Published: July 3, 2014

This is an open-access article distributed under the terms of the Creative Commons Attribution License, which permits unrestricted use, distribution, and reproduction in any medium, provided the original author and source are credited.

ABSTRACT:

Background: Helicobacter pylori (H. pylori) is the most important gastric carcinogen. However, the mechanisms of $\boldsymbol{H}$. pylori induced gastric carcinogenesis through STAT3 activation are largely unknown. We evaluated the effects of $H$. pylori infection on STAT3 activation and dissected the signalling network of STAT3 in $\boldsymbol{H}$. pylori-infected gastric carcinogenesis.

Methods: The expression of phospho-STAT3 (pSTAT3) was evaluated by immunohistochemistry and western blot. Gene expression array and chromatin immunoprecipitation were used to dissect the STAT3 signalling network on $H$. pylori co-cultured AGS.

Results: pSTAT3 was significantly higher in $\boldsymbol{H}$. pylori-positive gastritis than in $\boldsymbol{H}$. pylori-negative gastritis $(P=0.003)$. In addition, $98 \%$ of $H$. pylori positive intestinal metaplasia specimens showed STAT3 activation, whereas PSTAT3 was significantly decreased in all 43 specimens one year after $H$. pylori eradication $(P<0.001)$. Moreover, PSTAT3 was only detected in the $H$. pylori-infected gastric tissues of mice but not in control mice. We further identified 6 candidates (BRUNOL4, FGFR1, SHOX2, JAK3, MAPK8, and PDPN) were directly up-regulated by $H$. pylori induced STAT3 activation.

Conclusion: $\boldsymbol{H}$. pylori infection triggers the activation of STAT3 and de-regulates multitude of tumorigenic genes which may contribute to the initiation and progression of gastric cancer.

\section{INTRODUCTION}

Infection with the gram-negative bacterium Helicobacter pylori (H. pylori) is a major risk factor for gastric carcinoma. The cytotoxin-associated antigen A (CagA) gene codes for one of the H. pylori virulence proteins. Epidemiological studies reveal that CagA positive $H$. pylori strains are most closely related with an increased risk of gastric carcinogenesis in comparison with $H$. pylori CagA negative strains $[1,2]$. The CagA+
H. pylori strains infection could increase signal transducer and activator of transcription 3 (STAT3) and mitogenactivated protein kinase (ERK1/2) activation in $H$. pylori-dependent gastritis [3]. It has been also showed that activation of STAT3 pathway is associated with progression to gastric cancer [4]. These findings suggest that $H$. pylori infection interfere with STAT3 proteins activation in gastric epithelial cells, which may favour their long term colonization in the host stomach.

STAT3, a cytoplasmic signalling protein and nuclear 
transcription factor, is frequently found to be overactivated in a variety of human malignancies. Persistent STAT3 signalling could promote the growth and survival of tumor cells, induce tumor angiogenesis and suppress the anti-tumor immune responses [5, 6]. Targeted deletion of STAT3 was shown to prevent epithelial cancer [7] and may be a novel approach in cancer therapy [8]. Constitutive activation of STAT3 has also been demonstrated in human gastric cancer cell lines and in primary human gastric cancers [3, 9]. STAT3 expression strongly correlated with VEGF expression and microvessel density in human gastric cancer [10]. Moreover, transfection of dominant negative STAT3 or inhibition of STAT3 by AG490 in human gastric cancer cell lines resulted in reduced cell growth [9]. These data suggest an important role of STAT3 in the pathogenesis of human gastric cancer. However, the mechanisms of $H$. pylori induced STAT3 signalling network in gastric carcinogenesis are still largely unknown. In this study, we aim to evaluate the effect of H. pylori infection on STAT3 activation and to dissect the signalling network of STAT3 in the $H$. pylori-infected gastric carcinogenesis.

\section{RESULTS}

\section{STAT3 was activated in $H$. pylori-infected AGS cells}

To elucidate whether infection of virulence $H$. pylori trigers the STAT3 activation in GC cells in vitro, we co-cultured the GC cell lines AGS with $\mathrm{CagA}+H$. pylori strain and CagA- H. pylori strains respectively. Remarkably, treatment of AGS cells with CagA+ ATCC43504, but not the isogenic CagA mutant strains, resulted in a pronounced increasing in phosphorylation of STAT3 (pSTAT3) at Tyr705 (Figure 1A). Therefore, AGS and ATCC435040 were used for further experiments.

We next performed a time/H. pylori MOI course study to monitor the pSTAT3 level at different time points after $H$. pylori ATCC43504 infection. We observed that H. pylori increased the pSTAT3 by nearly 2 -fold at 0.5 hr in AGS cells; the pSTAT3 level was regulated in a time-dependent manner (Figure 1B). However, we did not observe a clear difference on the pSTAT3 level in response to different $H$. pylori MOIs (Figure 1B). Taken together, we established $H$. pylori strain ATCC43504 and AGS coculture model. Our findings demonstrated that pSTAT3 was induced by CagA positive H. pylori in AGS.

\section{pSTAT3 was increased in $H$. pylori-associated gastric tissues}

To investigate the effect of $H$. pylori on STAT3 activation in human gastric tissues, we examined the changes in pSTAT3 protein level on clinical specimen using immunohistochemistry (Figure 2A). The pSTAT3 staining analysis was summarized in Table 1 . We found that the expression of the active form of STAT3 was significantly higher in $H$. pylori-positive gastritis $(73.5 \%$, $61 / 83)$ than in $H$. pylori-negative gastritis $(50 \%, 35 / 70)(P$ $=0.003)$. To further confirm the connection between $H$. pylori infection and STAT3 activation, we measured the pSTAT3 level on 44 paired gastric intestinal metaplasia biopsies taken from $H$. pylori infected individuals before and one year after $H$. pylori eradication. We found that 98\% (43/44) of $H$. pylori positive intestinal metaplasia specimens showed positive staining for pSTAT3 before eradication, whereas therapy-based eradication of $H$. pylori significantly decreased pSTAT3 expression in all the $43 \mathrm{H}$. pylori-positive intestinal metaplasia patients

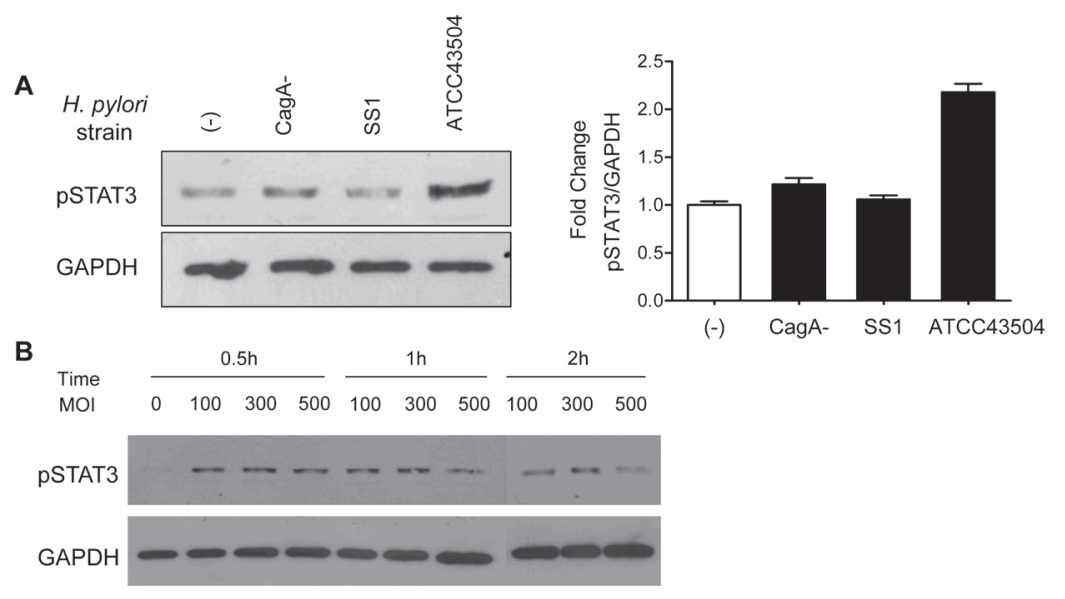

Figure 1: STAT3 was activated in $\boldsymbol{H}$. pylori ATCC43504 infected AGS cells. (A) AGS cells were treated with CagA $+H$. pylori strain ATCC43504 and CagA- H. pylori strains respectively. Whole cell lysates were analysed by immunoblotting with anti-pSTAT3 (Tyr705) antibody. The pSTAT3 protein band intensities were quantified and normalized to GAPDH intensities (right panel). (B) The expression of pSTAT3 was induced in AGS after treatment with ATCC43504 at various MOI and co-culture time. 
(Table 2, Figure 2B, $P<0.001$ ). These data provided direct evidence that $H$. pylori contributed to the activation of STAT3 in gastritis and intestinal metaplasia. In addition, pSTAT3 expression was detected in 27 human $H$. pylori positive gastric cancers using immunohistochemistry. pSTAT3 displayed stronger nuclear staining in the gastric cancer specimens compared with the adjacent normal tissues (Figure 2C).

A

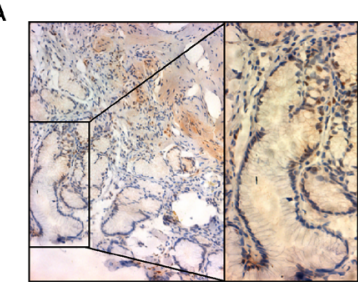

B H. pylori negative gastritis

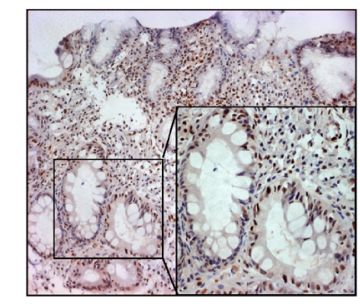

C
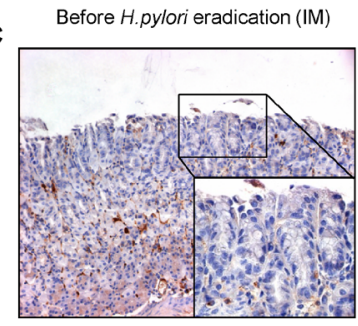

D

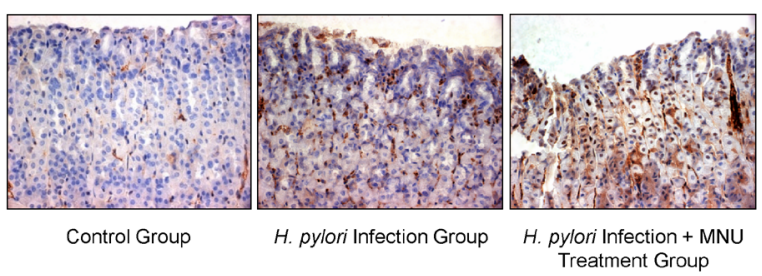

Figure 2: pSTAT3 was increased in $\mathrm{H}$. pylori-associated clinical gastric tissues. (A) Representative images of immunohistochemical staining of pSTAT3 in $H$. pylori negative gastritis, and in $H$. pylori positive cases. (B) Representative images of immunohistochemical staining of pSTAT3 in $H$. pylori infected intestinal metaplasia (IM) before and one year after eradication. (C) Representative images of immunohistochemical staining of pSTAT3 in gastric cancer tumor tissues and adjacent normal tissues. Original magnification, $\times 200$ (low power); $\times 400$ (high power). (D) pSTAT3 was increased in $H$. pylori SS1 infection mouse model. Representative images of immunohistochemical staining of pSTAT3 in $H$. pylori SS1 infection mouse model with or without MNU treatment. Original magnification, $\times 200$.
Table 1: Expression of pSTAT3 in $H$. pylori negative and $H$. pylori positive gastritis

\begin{tabular}{|c|c|c|c|c|c|}
\hline & & pSTAT3 & & & \\
\hline & No. & Negative & Positive & $x^{2}$ & $P$ Value \\
\hline $\begin{array}{l}H . \text { pylori } \\
\text { negative } \\
\text { gastritis }\end{array}$ & 70 & $35(50 \%)$ & $35(50 \%)$ & & \\
\hline $\begin{array}{l}\text { H. pylori } \\
\text { positive } \\
\text { gastritis }\end{array}$ & 83 & $\begin{array}{l}22 \\
(26.5 \%)\end{array}$ & $\begin{array}{l}61 \\
(73.5 \%)\end{array}$ & 8.97 & 0.003 \\
\hline
\end{tabular}

The expression of pSTAT3 was increased in $\mathrm{H}$. pylori infection mouse model

The association between $H$. pylori infection and pSTAT3 expression was further evaluated in mouse model with or without $H$. pylori Sydney Strain 1 (SS1) infection. We found that pSTAT3 was only detected in the $H$. pyloriinfected gastric tissues of mice $(2 / 3,66.7 \%)$, but not in those of the control mice (Table 3 ), providing further evidence that $H$. pylori infection stimulated the activation of STAT3 in the stomach (Figure 2D). Moreover, treatment of $H$. pylori infected mice with N-methyl-Nnitrosourea (MNU), an alkylating agent that induces gastric carcinogenesis in mouse, resulted a pronounced STAT3 activation in all the mice tested (Table 3, 100\%, $P$ $=0.005$ ). Thus, this in vivo study recapitulated our cellular and clinical findings, and demonstrated directly that $H$. pylori induced STAT3 activation which contributing to the gastric carcinogenesis.

\section{STAT3 promoted GC cell growth}

The activation of STAT3 after $H$. pylori infection led us to speculate that enhanced STAT3 activation contributed to the proliferative phenotype of the GC cells. Thus, we transfected pCDNA3.1-STAT3 into GC cell line MKN28 and AGS (Figure 3A) and cell variability was measured by MTT assay and colony formation assay. As expected, MKN28 cells with up-regulated STAT3 grew significantly faster than did control cells (Figure 3B). In addition, overexpression of STAT3 in AGS and MKN28 significantly promoted the colony growth (Figure $3 \mathrm{C}$ and 3D).

\section{Identification of STAT3 associated transcriptional target genes and pathways in $H$. pylori-infected AGS}

To assess the STAT3 associated transcriptional alterations induced by $H$. pylori infection, we performed genome expression microarrays on the ATCC43504- 
Table 2: pSTAT3 immunohistochemistry in 44 cases with paired intestinal metaplasia gastritis before and after $H$. pylori eradication

\begin{tabular}{|l|l|l|l|l|l|}
\hline & \multirow{2}{*}{ No. } & \multicolumn{2}{|l|}{$\mathrm{pSTAT3}$} & \multirow{2}{*}{$\chi 2$} & \multirow{2}{*}{$P$ Value } \\
\cline { 3 - 5 } & & Negative & Positive & & \\
\hline Before & 44 & $1(2.3 \%)$ & $43(97.7 \%)$ & 84.09 & $<0.001$ \\
\hline After & 44 & $44(100 \%)$ & $0(0 \%)$ & & \\
\hline
\end{tabular}

infected and non-infected AGS. AGS cells treated with either ATCC 43504 at $100 \mathrm{MOI}$ or PBS for $0.5 \mathrm{hr}$ were subjected to microarrays. Analysis of the microarray data for the two groups revealed a total of 849 genes with expression changes, among which 813 genes showed increased expressions in $H$. pylori-infected AGS with STAT3 activation and 36 genes showed decreased expressions (Figure 4A). These candidates with fold change $\geq 2$ (53 candidates up-regulated in H. pyloriinfected AGS) were subjected to gene ontology and pathway analysis. KEGG pathway analysis showed that the dysregulated genes were enriched in 11 different cancer pathways including ligand-receptor interaction, calcium signalling pathway, cell differentiation and MAPK signalling pathway (Figure 4B). Gene ontology analysis revealed significant up-regulation of genes with prominent roles in multiple cellular processes including ion binding, signal transduction and transporter activity (Figure 4C). Nine potential targets up-regulated in the expression microarray (BRUNOL4, FGFR1, SHOX2, JAK3, MAPK8, PDPN, IL10RA, MMP28, and TPPP8) were selected for further real-time PCR validation. We
Table 3: Expression of pSTAT3 in $\mathrm{H}$. pylori associated mouse model

\begin{tabular}{|l|l|l|l|l|l|}
\hline & \multirow{2}{*}{ No. } & \multicolumn{2}{|l|}{ pSTAT3 } & \multirow{2}{*}{$\chi^{2}$} & $\begin{array}{l}P \\
\text { Value }\end{array}$ \\
\cline { 3 - 5 } & Negative & Positive & & \\
\hline $\begin{array}{l}\text { Control } \\
\text { group }\end{array}$ & 4 & $4(100 \%)$ & $0(0 \%)$ & & \\
\hline $\begin{array}{l}\text { H. pylori } \\
\text { infection } \\
\text { group }\end{array}$ & 3 & $1(33.3 \%)$ & $2(66.7 \%)$ & 3.733 & 0.053 \\
\hline $\begin{array}{l}\text { H. pylori } \\
\text { infection } \\
+\quad \text { MNU } \\
\text { treatment } \\
\text { group }\end{array}$ & 4 & $0(0 \%)$ & $4(100 \%)$ & 8.0 & 0.005 \\
\hline
\end{tabular}

found that these candidates were significantly increased at transcriptional level upon infection of $H$. pylori in AGS (Figure 4D).

\section{Identification of direct STAT3 transcriptional target genes in $\mathrm{H}$. pylori-infected AGS cells}

To identify key tumorigenic-regulators directly controlled by STAT3 binding after $H$. pylori infection, we next performed ChIP on ATCC43504 infected AGS cells. After cross-linked with formaldehyde, DNA was sonicated into small fragments and immunoprecipitated by anti-pSTAT3 (Tyr705) polyclonal antibody. Conventional real-time PCR and ChIP-PCR analysis validated that 6 out of the 9 potential pSTAT3 (Tyr705) targets (BRUNOL4, FGFR1, SHOX2, JAK3, MAPK8 and PDPN) showed strong enrichment for pSTAT3 antibody but not the IgG
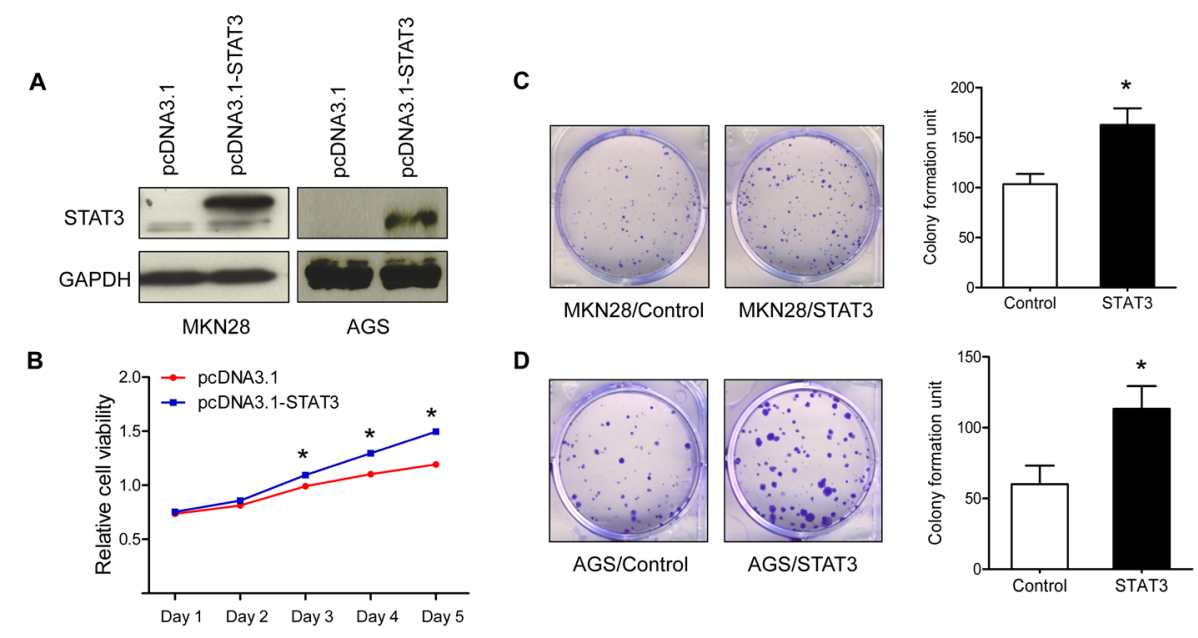

D
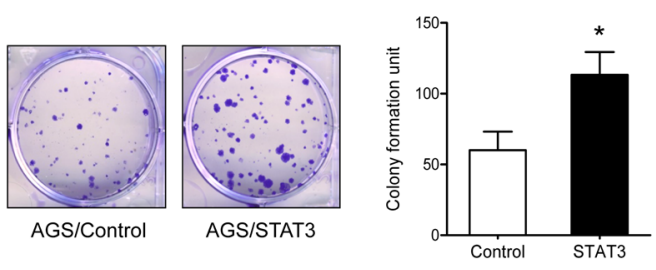

Figure 3: STAT3 processes oncogenic function in gastric cancer cells. (A) Ectopic expression STAT3 in AGS and MKN28. (B) Ectopic expression STAT3 promoted MKN28 cell growth. (C) Colony formation assay of STAT3-expressing or control MKN28 cells. (D) Colony formation assay of STAT3-expressing or control AGS cells Representative images of colonies formed are shown. * $\mathrm{P}<0.05$. 
control (Figure 4E). Collectively, our data suggested that the 6 candidates could be the potential tumorigenic genes that directly regulated by activated STAT3 in response to H. pylori infection.

\section{DISCUSSION}

In our study, we established a gastirc cancer cell line-H. pylori co-culture model and demonstrated that H. pylori pronouncedly induced STAT3 activation in GC cell line AGS after co-culture in vitro. By using a larger clinical sample cohort, we observed a correlation between STAT3 activation and $H$. pylori infection. We found an increased pSTAT3 level in the H pylori positive gastritis patients (Table 1). As shown in Table 2, we also found that STAT3 activation was significant reduced in the intestinal metaplasia one year after $H$. pylori eradication as compared with their initial $H$. pylori positive intestinal metaplasia specimen from 44 patients $(P<0.001)$. These data suggests that $H$. pylori infection and its related STAT3 activation play the pivotal role in the process of gastric inflammation to intestinal metaplasia, an important part of a progressive process of gastric cancer. Moreover, we found that pSTAT3 displayed strongly nuclear staining in the $H$. pylori positive gastric cancer specimen, whereas the pSTAT3 staining was relatively weak in the adjacent normal stomach. Thus, based on these clinical specimens, we found that pSTAT3 was significantly associated with $H$. pylori-positive gastritis, $H$. pylori-positive intestinal metaplasia and gastric cancer. In mice, we observed that expression of pSTAT3 was increased in response to H. pylori infection, and up-regulated remarkably in a MNU induced gastric cancer. Collectively, our findings suggested that STAT3 activation in response to H. pylori infetion contributed to the initiation of gastric carcinogenesis.

We also demonstrated the enhanced STAT3 expression contributed to the proliferative phenotype of the AGS and MKN28 cell lines. Upon activation, pSTAT3 translocates to cell nucleus and mediates the expression downstream effector genes, leading to cell survival, proliferation, angiogenesis, invasion and migration [11]. Using gene expression microarray, we identified that totally 849 genes were dysregulated in AGS after $H$. pylori infection. The dysregulated genes were enriched in 11 different cancer pathways including ligand-receptor interaction, calcium signalling pathway, cell differentiation and MAPK signalling pathway (Figure 4B) with an important implication for the H. pyloriinduced gastric tumorigenesis (Figure 4C). Elucidating the direct downstream genes of STAT3 in response to H. pylori infection is important for unrevealing critical molecular events of gastric carcinogenesis. Using gene expression microarray coupled with ChIP-PCR assays, we identified 6 candidate genes (BRUNOL4, FGFR1, $S H O X 2, J A K 3, M A P K 8$, and $P D P N)$ that directly upregulated by $H$. pylori induced STAT3 activation (Figure 4E). Among these candidates, BRUNOL4 and FGFR1 are the two novel oncogenic candidates that may play roles in gastric carcinogenesis. Bruno-like 4 splicing factor (BRUNOL4, also known as CELF4) belongs to the CELF/BRUNOL protein family which involved in post-

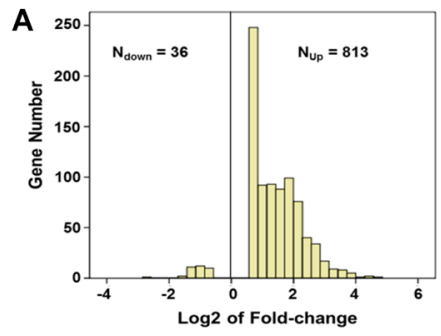

B Gene analysis with KEGG pathway

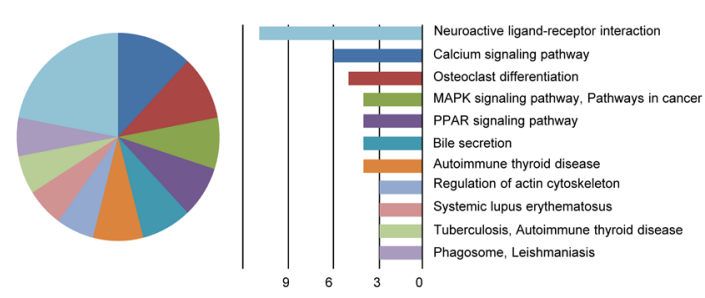

D

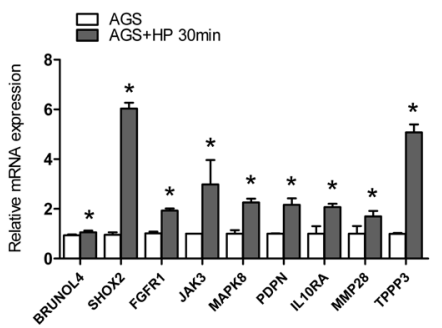

C Gene analysis with Molecular Function

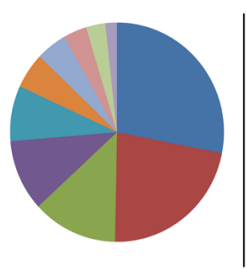

$\mathbf{E}$

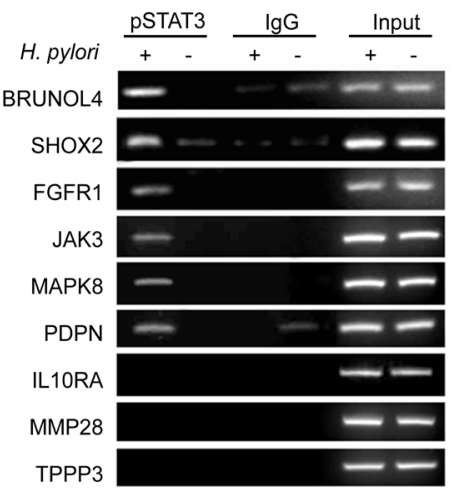

Figure 4: Identification of key tumorigenic-regulators directly controlled by STAT3 following $\boldsymbol{H}$. pylori infection. (A) 849 genes were differently expressed in ATCC43504-infected AGS relative to control group. (B) Top 11 dysregulated pathways using the KEGG database. (C) Gene ontology of the cDNA microarray. (D) Quantitative RT-PCR validation of the 9 potential targets in AGS cells treated with ATCC43504 for 30 min relative to the untreated cells. GAPDH was used as an internal control. (E) Confirmation of the potential pSTAT3 target genes by ChIP-PCR using anti-pSTAT3 (Tyr705) antibody or irrelevant antibody against IgG (negative control) on ATCC43504 infected and non-infected AGS cells. Input represents the genomic DNA. ${ }^{*} P<0.05$. 
transcriptional gene expression processes, including premRNA alternative splicing and editing. [12]. Although the function of BRUNOL4 in gastric cancer development is still largely unknown, Rouzier reported that the expression of BRUNOL4 was associated with paclitaxel sensitivity in breast cancer [13]. FGFR1 is a member of the fibroblast growth factor receptor family, which interacts with its ligand fibroblast growth factors (FGF) to induce a cascade of downstream signalling that regulates cell proliferation, survival, migration and differentiation [14]. The potent oncogenic potential of the FGFR1 has been confirmed by transgenic mouse study where Fgfr1 express in the mammary epithelium, resulting in enhanced mammary tumorigenesis [15]. In human, gene amplification of FGFR1 in $22 \%$ of squamous cell lung cancer [16], and $10 \%$ of ER-positive breast cancer [17].

In conclusion, our results indicated that $H$. pylori infection triggers the activation of STAT3 to de-regulate a multitude of tumorigenic genes which may contribute to the initiation and transformation of gastric carcinogenesis through mediating the downstream effectors and signalling pathways of STAT3.

\section{METHODS}

\section{Cell culture}

The gastric cancer cell lines AGS and MKN28 were purchased from the American Type Culture Collection (ATCC, Manassas, VA) and maintained in RPMI 1640 growth medium, supplied with $10 \%$ fetal bovine serum, 50 $\mathrm{IU} / \mathrm{mL}$ penicillin, $50 \mathrm{ug} / \mathrm{mL}$ streptomycin (All Invitrogen, Carlsbad, CA). Cells were kept at $37^{\circ} \mathrm{C}$ in a humidified incubator with $5 \% \mathrm{CO}_{2}$.

\section{H. pylori culture and in vitro infection model}

H. pylori strains (SS1, CagA-, ATCC43504 CagA+) were inoculated on Brucella agar plates containing 5\% sheep blood and incubated at $37{ }^{\circ} \mathrm{C}$ for 3-4 days under microaerophilic conditions in a humidified $\mathrm{CO}_{2}$ incubator (Thermo Fisher Scientific, Wilmington, DE). The plate culture was then suspended and $H$. pylori were incubated with gastric cancer cells for the indicated incubation period $(0.5,1$, and $2 \mathrm{hr})$. The cells were then washed three times with PBS and harvested.

\section{Patients and gastric biopsies}

Gastric biopsies were collected from primary $H$. pylori associated chronic gastritis ( 83 cases) and gastric cancer (27 cases) during endoscopy according to a standard protocol, prior to any therapeutic intervention.
We also rescued 44 paired gastric biopsies taken from H. pylori infected individuals before and one year after eradication. Moreover, gastric biopsies were also collected from $H$. pylori negative gastritis (70 cases) as controls. Patients were excluded if they had other cancers or inflammation diseases. All patients and controls gave informed consent, and the study protocol was approved by the Clinical Research Ethics Committee of the Chinese University of Hong Kong.

\section{H. pylori infection in mouse model}

A total of 11 male C57BL/6 mice (6-8-week old) were obtained from the Animal Service Centre at the Chinese University of Hong Kong. All of the mice were given a standard pellet chow diet and autoclaved distilled water ad libitum and were maintained in specific pathogen-free conditions. MNU (240 p.p.m. SigmaAldrich, St Louis, MO) was prepared freshly twice a week by dissolving in distilled water. The solutions were administered ad libitum for a total 5 cycles of 1-week regimen followed by 1 -week pause, as drinking water in light-shielded bottles. The H. pylori SS1 were used to infect the mice. After $48 \mathrm{hr}$ of fasting, $0.1 \mathrm{~mL}$ suspension of $H$. pylori containing $1 \times 10^{9}$ colony-forming units $(\mathrm{CFU})$ $/ \mathrm{mL}$ were delivered to mice intragastrically, triple times every other day within a week. The mice were sacrificed 40 weeks after $H$. pylori infection. Animal studies were performed in accordance with guidelines approved by the Animal Experimentation Ethics Committee of the Chinese University of Hong Kong.

\section{Chromatin immunoprecipitation (ChIP)}

ChIP was performed as described previously [18]. Briefly, $1 \times 10^{8}$ AGS cells treated with ATCC43504 multiplicity of infection (MOI) of 100 in serum free RPMI1640 medium for $0.5 \mathrm{hr}$ were crosslinked with $1 \%$ formaldehyde for 10 minutes at room temperature and quenched by glycine. After cell lysis, the chromatin was fragmented into 100 - 500 bp by Bioruptor Sonicator (Diagenode, Denville, NJ) and protein-DNA complexes were immunoprecipitated (IP) by 5 ug anti-pSTAT3 (Tyr 705) antibody (Cell Signaling Technology, Beverly, MA) or anti-IgG antibody (Sigma-Aldrich) Dynal magnetic bead (Invitrogen) mix on rotator at $4^{\circ} \mathrm{C}$ overnight. After washing and reversal of crosslinks, the IP and input DNA were purified and subjected to PCR.

\section{Gene expression microarray analysis}

Gene expression profiles of AGS and $H$. pylori infected AGS cells were analyzed using Whole Human Genome Microarray Kit, 4x44K (Agilent Technologies, 
Palo Alto, CA), which contained 19,596 cDNA clones. Signal intensities were analyzed using a GenePix 4000A scanner (Axon Instruments, Molecular Devices Crop., Palo Alto, CA). Array data were presented as log base 2-ratio of the $\mathrm{Cy} 5 / \mathrm{Cy} 3$ signals.

\section{Conventional and quantitative ChIP-PCR assays}

For target genes validation, PCR primers targeting a region within $150 \mathrm{bp}$ of the putative binding site were designed to detect IP and input DNA. $2 \mathrm{uL}$ IP and 2\% input DNA were used as a template for conventional PCR assay. For quantitative ChIP-PCR, equal amounts of IP and diluted input DNA were used for Power SYBR Greenbased detection (Applied Biosystems, Grand Island, NY).

\section{Western blot}

Protein lysates from cell lines were prepared using protease inhibitor cocktail-containing (Roche, Basel, Switzerland) lysis buffer. Protein concentration was determined by the Bradford method (Bio-Rad Laboratories, Hercules, CA). Antibody-antigen complexes were detected using the ECL Plus Western Blotting Detection Reagents (GE Healthcare Piscataway, NJ). Primary antibodies used were rabbit anti-STAT3 (Cell Signaling Technology, Boston, MA), rabbit anti-pSTAT3Try705 (Cell Signaling Technology), mouse anti-GAPDH (Santa Cruz Biotechnology, Santa Cruz, CA).

\section{Immuhistochemistry}

Five um sections from formalin-fixed paraffinembedded archive tissues were stained with polyclonal antibody against pSTAT3 (1:25). Antibody was incubated at room temperature for $2 \mathrm{hr}$, and chromogen development was performed using the universal HRP Multimer Ultraview Kit on Benchmark XL (Ventana Medical System, Oro Valley, AZ). The nuclear expression was assessed by a proportion score of the positive tumor cells and was categorized into negative (extensity $<10 \%$ ), positive (extensity $\geq 10 \%$ ). The scoring was independently assessed by 2 investigators.

\section{Colony formation assay and cell viability assay}

GC cell lines were transfected with pcDNA3.0STAT3 or pcDNA3.0 using lipofectamine 2000 (Invitrogen) and selected with G418 at $0.5 \mathrm{mg} / \mathrm{ml}$ (Calbiochem, Darmstadt, Germany) for 14 days. The resulting cells were then fixed with $70 \%$ ethanol and stained with $5 \%$ crystal violet, and individual colonies with more than 50 cells were counted. Cell viability was determined using Vybrant MTT Cell Proliferation Assay
Kit (Invitrogen).

\section{Statistical Analysis}

The results were expressed as mean \pm standard deviation (SD). Mann-Whitney U test was performed to compare the variables of the two sample groups. The association between patient characteristics and pSTAT3 status was analyzed by the $\chi^{2}$ test. All analyses were performed using SAS for Windows software, version 9 (SAS Institute). All tests of statistical significance were two-sided. $P$-values less than 0.05 were considered statistically significant.

\section{ACKNOWLEDGMENTS}

This study was supported by research funds of Hong Kong RFCID (10090942), China 973 Program (2010CB529305), China 863 Program (2012AA02A203), and Shenzhen Municipal Science and Technology R\&D funding (JCYJ20120619152326450), Shenzhen Virtual University Park Support Scheme to CUHK Shenzhen Research Institute.

\section{Author contribution}

JZ performed the experiments, analyzed data and drafted the manuscript; YD analyzed data and wrote the manuscript, WK, MYG, JHMT, EKWN, PWYC and ASLC assisted with performing experiments and providing materials; KFT and JJYS comment on the study; JY designed and supervised the project, analyzed data and revised the manuscript.

\section{Authors' declaration of personal interests}

The authors disclosed no potential conflicts of interest.

\section{REFERENCES}

1. Cabral MM, Mendes CM, Castro LP, Cartelle CT, Guerra J, Queiroz DM, Nogueira AM. Apoptosis in Helicobacter pylori gastritis is related to cagA status. Helicobacter. 2006; 11: 469-476.

2. Calvet X, Ramírez Lázaro MJ, Lehours P, Mégraud F. Diagnosis and epidemiology of Helicobacter pylori infection. Helicobacter. 2013; 18 Suppl 1: 5-11.

3. Jackson CB, Judd LM, Menheniott TR, Kronborg I, Dow C, Yeomans ND, Boussioutas A, Robb L, Giraud AS. Augmented gp130-mediated cytokine signalling accompanies human gastric cancer progression. J Pathol. 2007; 213: 140-151. 
4. Bronte-Tinkew DM, Terebiznik M, Franco A, Ang M, Ahn D, Mimuro H, Sasakawa C, Ropeleski MJ, Peek RM Jr, Jones NL. Helicobacter pylori cytotoxin-associated gene A activates the signal transducer and activator of transcription 3 pathway in vitro and in vivo. Cancer Res. 2009; 69: 632639.

5. Yu H, Jove R. The STATS of cancer - New molecular targets come of age. Nature Rev Cancer. 2004; 4: 97-105.

6. Judd LM, Bredin K, Kalantzis A, Jenkins BJ, Ernst M, Giraud AS. STAT3 activation regulates growth, inflammation, and vascularization in a mouse model of gastric tumorigenesis. Gastroenterology. 2006; 131: 10731085.

7. Chan KS, Sano S, Kiguchi K, Anders J, Komazawa N, Takeda J, DiGiovanni J. Disruption of Stat3 reveals a critical role in both the initiation and the promotion stages of epithelial carcinogenesis. J Clin Invest. 2004; 114: 720728.

8. Darnell JE. Validating Stat3 in cancer therapy. Nature Med. 2005; 11: 595-596.

9. Kanda N, Seno H, Konda Y, Marusawa H, Kanai M, Nakajima T, Kawashima T, Nanakin A, Sawabu T, Uenoyama Y, Sekikawa A, Kawada M, Suzuki K, et al. STAT3 is constitutively activated and supports cell survival in association with survivin expression in gastric cancer cells. Oncogene. 2004; 23: 4921-4929.

10. Gong W, Wang L, Yao JC, Ajani JA, Wei D, Aldape KD, Xie K, Sawaya R, Huang S. Expression of activated signal transducer and activator of transcription 3 predicts expression of vascular endothelial growth factor in and angiogenic phenotype of human gastric cancer. Clin Cancer Res. 2005; 11: 1386-1393.

11. Yu H, Pardoll D, Jove R. STATs in cancer inflammation and immunity: a leading role for STAT3. Nat Rev Cance.r 2009; 9: 798-809.

12. Kino Y, Washizu C, Oma Y, Onishi H, Nezu Y, Sasagawa $\mathrm{N}$, Nukina $\mathrm{N}$, Ishiura $\mathrm{S}$. MBNL and CELF proteins regulate alternative splicing of the skeletal muscle chloride channel CLCN1. Nucleic Acids Res. 2009; 37: 6477-6490.

13. Rouzier R, Rajan R, Wagner P, Hess KR, Gold DL, Stec J, Ayers M, Ross JS, Zhang P, Buchholz TA, Kuerer H, Green M, Arun B, et al. Microtubule-associated protein tau: a marker of paclitaxel sensitivity in breast cancer. Proc Natl Acad Sci U S A. 2005; 102: 8315-8320.

14. Grose R, Dickson C. Fibroblast growth factor signaling in tumorigenesis. Cytokine Growth Factor Rev. 2005; 16: 179186.

15. Pond AC, Herschkowitz JI, Schwertfeger KL, Welm B, Zhang Y, York B, Cardiff RD, Hilsenbeck S, Perou CM, Creighton CJ, Lloyd RE, Rosen JM. Fibroblast growth factor receptor signaling dramatically accelerates tumorigenesis and enhances oncoprotein translation in the mouse mammary tumor virus-Wnt-1 mouse model of breast cancer. Cancer Res. 2010; 70: 4868-4879.
16. Weiss J, Sos ML, Seidel D, Peifer M, Zander T, Heuckmann JM, Ullrich RT, Menon R, Maier S, Soltermann A, Moch $\mathrm{H}$, Wagener P, Fischer F, et al Frequent and focal FGFR1 amplification associates with therapeutically tractable FGFR1 dependency in squamous cell lung cancer. Sci Transl Med. 2010; 2: 62ra93.

17. Courjal F, Cuny M, Simony-Lafontaine J, Louason G, Speiser P, Zeillinger R, Rodriguez C, Theillet C. Mapping of DNA amplifications at 15 chromosomal localizations in 1875 breast tumors: Definition of phenotypic groups. Cancer Res. 1997; 57: 4360-4367.

18. Feng H, Cheng AS, Tsang DP, Li MS, Go MY, Cheung YS, Zhao GJ, Ng SS, Lin MC, Yu J, Lai PB, To KF, Sung JJ. Cell cycle-related kinase is a direct androgen receptorregulated gene that drives $\beta$-catenin/T cell factor-dependent hepatocarcinogenesis. J Clin Invest. 2011; 121: 3159-3175. 